\title{
Traduire
}

Ine autre perspective sur I t tadaduction

Revue française de la traduction

$232 \mid 2015$

Intraduisible? Vous voulez rire !

\section{Traduire les jeux de mots et calembours de journaux satiriques - Le Canard enchaîné et Private Eye}

Joëlle Popineau

\section{(2) OpenEdition}

Journals

Édition électronique

URL : http://journals.openedition.org/traduire/694

DOI : 10.4000/traduire.694

ISSN : 2272-9992

Éditeur

Société française des traducteurs

Édition imprimée

Date de publication : 15 juin 2015

Pagination : 41-55

ISSN : 0395-773X

\section{Référence électronique}

Joëlle Popineau, «Traduire les jeux de mots et calembours de journaux satiriques - Le Canard enchaîné et Private Eye », Traduire [En ligne], 232 | 2015, mis en ligne le 15 juin 2017, consulté le 10 décembre 2020. URL : http://journals.openedition.org/traduire/694 ; DOI : https://doi.org/10.4000/ traduire.694 


\section{Traduire les jeux de mots et calembours de journaux satiriques - Le Canard enchaîné et Private Eye}

\section{Joëlle Popineau}

Comment faire pour traduire la Une d'un journal humoristique français en anglais ? Le contenu des jeux de mots est-il universel ? Quels en sont les procédés de création ? Sur quoi repose le rire que suscitent les titres ou rubriques des journaux?

Cet article se propose de passer en revue les différents types de jeux de mots dans les deux langues et d'en expliquer le mécanisme linguistique afin de proposer des pistes de traduction, une suggestion ou la bonne solution. Car traduire le jeu de mots, c'est le comprendre pour pouvoir le faire passer dans l'autre langue.

Traduire c'est aussi faire des choix de stratégies traductionnelles : le traducteur est partagé entre privilégier le lexique et une approche stylistico-linguistique ou privilégier le message et une approche fonctionnaliste. Ces enjeux théoriques sont détaillés et appliqués ici à des exemples issus de deux journaux, Le Canard enchaîné pour la langue française et Private Eye pour la langue anglaise.

\section{Méthodologie}

Notre méthodologie consiste à étudier certains titres du Canard enchaîné(1) et de Private Eye(2), à en analyser vocabulaire et références et à proposer des pistes de traduction.

Les titres choisis dans le Canard enchaîné proviennent de trois numéros :

$-n^{\circ} 4917$ du 21 janvier 2015,

$-n^{\circ} 4919$ du 4 février 2015,

$-n^{\circ} 4921$ du 18 février 2015 (voir l'illustration).

(1) Le courriel d'autorisation de " reproduire certains titres et extraits d'articles parus dans [I] es colonnes " date du 26 mars 2015 ; l'interlocuteur est Nicolas Brimo, responsable du service de rédaction.

(2) La Pressdam limited licence for reproduction en date du 26 février 2015 porte sur l'autorisation de reproduire les textes et articles des trois hors-série achetés en ligne énumérés ci-dessus et autres titres de la version en ligne. 
Les titres issus de Private Eye sont ceux des hors-série achetés en ligne :

- Shady Arabia and the Desert Fix (numéro 1375 du 2 octobre 2014);

- The Phone Hacking Scandal (numéro 1370 du 11 juillet 2014) ;

- Lockerbie - Private Eye's special report into the Lockerbie disaster (2001).

D'autres titres de Private Eye proviennent également du site web du journal (www.private-eye.co.uk).

\section{2. Êtes-vous sourcier ou cibliste(3) ?}

Une approche traductionnelle est dite sourcière lorsqu'elle se focalise sur le texte en langue source (LS) ; l'approche cibliste, quant à elle, se différencie par l'intérêt porté au texte et son examen en langue cible (LC).

Ces théories s'opposent depuis quelques décennies dans le domaine des sciences de la traduction et sont à l'origine d'une querelle entre deux groupes de linguistes-traductologues. Ainsi, et de façon très schématique, cette dichotomie sourcier / cibliste se décline en paires antinomiques : traduction universitaire / traduction professionnelle, traduction littéraire / traduction pragmatique, traduction fidèle / traduction infidèle, approche stylistico-linguistique / approche fonctionnaliste, où chacun choisira son camp.

\subsection{Approche sourcière}

L'approche stylistique sourcière avec laquelle nous avons choisi de travailler est celle décrite par Vinay et Darbelnet (1958) dans Stylistique comparée du français et de l'anglais. Sept procédés de traduction sont énumérés comme autant de mécanismes de traduction d'un énoncé du français vers l'anglais et vice et versa : l'emprunt, le calque, la traduction littérale, la transposition, la modulation, l'équivalence et l'adaptation sont ainsi décrits et illustrés par de nombreux exemples (tableau 1).

(3) Le livre de Jean-René Ladmiral éclaire et illustre ce schisme de façon détaillée et plaisante : Sourcier ou cibliste (2014), Traductologiques : Les Belles Lettres. 
Tableau 1 - Procédés de traduction et quelques exemples - Vinay et Darbelnet

\begin{tabular}{|c|c|}
\hline Nom du procédé & Exemples en français et anglais \\
\hline $\begin{array}{l}\text { Emprunt } \\
\text { (mot pris tel quel) }\end{array}$ & $\begin{array}{l}\text { Nous allons au pub } \\
\text { We will rendezvous at eight }\end{array}$ \\
\hline $\begin{array}{l}\text { Calque } \\
\text { (mot traduit) }\end{array}$ & $\begin{array}{l}\text { The White House - la Maison Blanche } \\
\text { Le Secrétaire d'État américain - the US Secretary of State }\end{array}$ \\
\hline $\begin{array}{l}\text { Traduction littérale } \\
\text { (mot à mot) }\end{array}$ & What time is it? Quelle heure est-il ? \\
\hline $\begin{array}{l}\text { Transposition } \\
\text { (syntaxe modifiée) }\end{array}$ & $\begin{array}{l}\text { L'enfant a traversé la Loire à la nage - The child swam across the Loire } \\
\text { II se peut que je vienne - I may come }\end{array}$ \\
\hline $\begin{array}{l}\text { Modulation } \\
\text { (point de vue modifié) }\end{array}$ & $\begin{array}{l}\text { Propriété interdite - No trespassing } \\
\text { I am in no rush - J'ai le temps }\end{array}$ \\
\hline $\begin{array}{l}\text { Équivalence } \\
\text { (formes idiomatiques) }\end{array}$ & It never rains but it pours - Un malheur n'arrive jamais seul \\
\hline $\begin{array}{l}\text { Adaptation } \\
\text { (éléments culturels) }\end{array}$ & $\begin{array}{l}\text { I'Hexagone - France } \\
\text { before you can say Jack Robinson - en un clin d'œil }\end{array}$ \\
\hline
\end{tabular}

Cette approche a été actualisée par les travaux de Chuquet et Paillard (1989) : Approche linguistique des problèmes de traduction(4). Les auteurs réduisent la liste des procédés syntaxiques aux procédés " centraux ", à savoir la transposition et la modulation, les autres procédés relevant selon eux du lexique (emprunt, calque, traduction littérale et équivalence) ou de facteurs socio-culturels (adaptation).

\subsection{Approche cibliste}

L'approche cibliste décrite dans cet article est l'approche fonctionnaliste de Christiane Nord (1997/2008) : La traduction : une activité ciblée. Introduction aux approches fonctionnalistes.

Cette approche - minoritaire dans les milieux universitaires français - propose d'envisager la fonction du document dans le processus de traduction : le principe de base est que toute traduction est motivée par un but précis, le postulat secondaire étant que le but initial du texte source peut différer du but initial du texte cible : par exemple, un acte de naissance rédigé en

(4) Chuquet et Paillard 1989, (1989) : p. 10. 
LS est une déclaration officielle de naissance d'une personne en tant qu'événement légal et administratif, alors que sa traduction en LC justifie de sa date de naissance et de sa nationalité en tant que fait administratif.

L'approche fonctionnaliste prend ainsi en compte l'objectif de la traduction, le support à traduire, le public visé par la traduction (lectorat) et les éléments culturels du pays de la LC.

Cette approche cibliste est courante dans le milieu professionnel, les traducteurs ayant une " conscience intuitive du fonctionnalisme".

Ces deux approches ne sauraient être hiérarchisées, ni prises indépendamment l'une de l'autre : la traduction étant l'art du compromis, notre compromis est tout aussi théorique.

\section{Les journaux étudiés}

Les deux journaux choisis pour notre étude offrent des points communs et quelques différences.

\subsection{Le Canard enchaîné}

Ce " journal satirique français paraissant le mercredi " a été créé le 10 septembre 1915 par Maurice Maréchal, rédacteur, et Henri-Paul Gassier, dessinateur. Ces deux hommes engagés écrivent pour s'attaquer "à la guerre, à la censure, aux politiciens, aux affairistes, aux curés, au pouvoir, à la guillotine " avec le rire pour seule arme. Le réseau de lecteurs est confidentiel(5) ; la censure, très présente, rendait quelquefois le journal illisible, tant il était troué de blancs(6). En riposte, les deux journalistes « mettent au point un langage codé : antiphrases, démentis qui valent confirmations, phrases à l'envers, etc., bref tout un arsenal qui fait du lecteur un initié, presque un complice(7) ॥. On retrouve toujours cette complicité entre lecteurs et chroniqueurs à l'heure actuelle.

Oscillant entre journal d'investigation et d'opposition pendant près de 60 ans, le Canard enchaîné a su dévoiler de grands scandales : I'“ Affaire des diamants de Giscard(8) " ou encore la publication de la feuille d'impôts de Marcel Dassault(9).

(5) Madame Maréchal livre le journal à pied chez les lecteurs au début de la parution en 1915.

(6) La censure est souvent personnifiée par Anastasie, une vieille dame imaginaire tenant une paire de ciseaux. (source : fr.wikipedia.org)

(7) Ibid.

(8) Le Canard du 10 octobre 1979 publie le fac-similé d'une commande de Bokassa 1er, ancien empereur de Centrafrique, datée de 1973, au Comptoir National du Diamant pour une plaquette de diamants de trente carats destinée à Valéry Giscard d'Estaing.

(9) Le Canard enchaîné du 11 septembre 1979 publie la feuille d'imposition de Marcel Dassault de 1978 selon laquelle il perçoit 24 millions de francs sans s'attribuer aucun salaire des entreprises qu'il détient. 
Ses rédacteurs en chef actuels sont Erik Emptaz et Louis-Marie Horeau et sa devise : "La liberté de la presse ne s'use que quand on ne s'en sert pas(10)".

Selon la rumeur, le journal disposerait de nouveaux pigistes, certains prenant part directement au Conseil des ministres, ou étant très proches des ministères et des partis politiques français, véritables " gorges profondes(11) " à la française : I'ancien porte-parole de l'UMP, Dominique Paillé, a reconnu publiquement en 2012 être un ex-informateur du Canard enchaîné. La rumeur prête aussi ce statut d'informateur à d'anciens hommes politiques devenus présidents ; enfin, le Canard dispose d'informateurs auprès de grands groupes industriels français, Renault pour ne citer qu'un exemple.

Certaines des grandes rubriques du Canard ont des titres sémantiquement liés à l'emblème du journal : le Canard ; on y trouve, entre autres:

- La Mare aux canards, section d'informations générales (p. 2 et 3),

- Minimares (p. 2),

- Le coin-coin des variétés (p. 7),

- Prises de bec, ou portrait d'un people (p. 7),

- Conflit de canard, rubrique sur la malbouffe (p. 7),

- Plouf, rubrique politico-économique (p. 7).

D'autres rubriques sont plus générales : La boîte aux images, Sur l'album de la Comtesse, Dans la presse déchaînée, etc.

Le Canard enchaîné, journal indépendant de toute rente publicitaire, est décrit comme l'un des journaux les plus rentables de France, sa seule source de revenus provenant de ses abonnements et de ses ventes en kiosque : il est tiré en moyenne à 477000 exemplaires papier par semaine en bichromie (noir et rouge écarlate).

Le Canard, longtemps épinglé comme anarchiste, se veut objectif et ne favorise actuellement ni la droite ni la gauche françaises.

Nous utiliserons indifféremment Canard ou Canard enchaîné pour désigner ce journal.

(10) II s'agit de la devise du Canard toujours imprimée en dernière page, remise à l'honneur lors des attentats de janvier 2015 en France.

(11) Deep Throat: pseudonyme utilisé par le mystérieux informateur du Washington Post à l'origine des révélations du Watergate qui allaient aboutir à la démission de Richard Nixon en 1974. 


\subsection{Private Eye}

Private Eye est un journal satirique bimensuel britannique appartenant au groupe Pressdam Limited, sis à Londres, dont le rédacteur en chef est lan Hislop, journaliste satirique. Créé en 1961, Private Eye, journal d'investigation, s'attaque à l'establishment, aux cercles politiques et aux personnalités publiques britanniques, mettant en exergue leur incompétence, inefficacité et vacuité, pointant du doigt les faits de corruption qui leur sont reprochés et reproduisant leurs propos pompeux ; Private Eye se définit comme a thorn in the side(12) of the British establishment.

Chaque numéro du journal tire à 225000 exemplaires(13) et son site internet permet de consulter les numéros en ligne par accès payant. Ses similitudes avec le Canard enchaîné sont nombreuses : le journal trouve ses sources auprès de ses nombreux informateurs et il joue, lui aussi, le rôle d'empêcheur de tourner en rond dans la sphère politique. Les rubriques de Private Eye sont également des détournements de mots ou de dictons : Street of Shame, Rotten Boroughs ou In the Back.

La popularité du journal fait que de nombreuses blagues et jeux de mots sont entrés dans la langue courante anglaise : par exemple, "Ugandan discussions(14) " est un euphémisme qui désigne une relation sexuelle pendant l'exercice de ses fonctions et toutes les expressions contenant "Uganda " ont dorénavant le même sens(15).

Nous utiliserons indifféremment Private Eye ou Eye pour désigner ce journal.

\section{Les procédés linguistiques visant à faire rire}

Différents procédés linguistiques permettant de produire des énoncés burlesques ont été régulièrement utilisés par les grands comiques ou virtuoses de la langue :

- la polysémie, procédé sémantique le plus communément utilisé, se retrouve ainsi dans toutes les histoires drôles ou blagues du monde : dans l'énoncé cohabitent plusieurs sens d'un mot donné, créant une double lecture et le rire ;

(12) N.d.T : " grain de sable empêchant le pouvoir établi de tourner en rond ", ou " empêcheur de tourner en rond ".

(13) Chiffres de 2013, selon http://www.pressgazette.co.uk/magazine-abcs-full-circulation-round-first-half-2013.

(14) L'histoire raconte qu'une journaliste aurait eu une entrevue privée avec un ancien ministre "à l'étage supérieur pour discuter de la situation en Ouganda " (upstairs discussing Uganda), prétexte donné par les deux impétrants.

(15) Une entrée de wikipedia est consacrée aux recurring in-jokes in Private Eye (source : http://en.wikipedia.org/wiki/Recurring_in-jokes_in_Private_Eye) 
- la contrepèterie, véritable jonglerie lexicale qui consiste à inverser des syllabes, est fréquente dans de nombreuses langues : ces spoonerisms produisent également des énoncés grivois chez nos voisins d'Outre-Manche et sont complétés par les kniferisms ou forkerisms (une définition de ces termes est donnée plus loin) ;

- le troisième procédé linguistique, plus complexe, est une combinaison des deux premiers : l'effet burlesque est atteint par déformation lexicale des expressions idiomatiques qui conservent toutefois leur " chaîne phonétique "; le dicton, le proverbe ou l'expression idiomatique français - avec sa rythmique propre - est ainsi déformé par insertion d'une ou plusieurs syllabes créant une double lecture et faisant passer un message décalé déclenchant le rire.

\subsection{Polysémie et jeux de mots ou wordplays}

Le Larousse définit le " jeu de mots " comme une " plaisanterie fondée sur la ressemblance des mots ". Celle-ci porte sur des homonymes, des homophones ou homographes. Cette plaisanterie est illustrée dans les exemples (1) et (2).

(1) Le pape exhorte les catholiques à ne pas se reproduire " comme des lapins". L'esprit Charlie déteint, voilà que le pape parle de lapines! (Le Canard enchaîné, $n^{\circ} 4917$ du mercredi 21 janvier 2015, p. 8).

où le jeu de mots repose sur la proximité phonétique entre le mot lapines, les femelles des lapins, et la pine, mot argotique pour désigner le sexe masculin ; le tout s'inscrit dans une référence à l'esprit du journal satirique et irrévérencieux, Charlie Hebdo, décimé par les attentats du 7 janvier 2015.

Dans (2), l'exemple d'homophonie est tiré de Private Eye(16) (édition en ligne du 30 mars) :

(2) Fifa gets ready for a big sheikh-up

où l'effet burlesque vient de la similitude phonétique entre shake-up et sheikh-up, qui fait référence au bouleversement radical (shake-up) et à la redistribution des cartes au sein de la Fédération internationale de Football Association (FIFA) provoqués par la possible élection d'un cheikh dans le comité directeur de ladite FIFA. Cet exemple est aussi appelé cacographie, orthographe fautive ou mauvais style(17).

Il existe des variantes aux jeux de mots : le kakemphaton(18) résulte d'un hasard malencontreux :

(3) Il a porté comme l'ensemble des joueurs une crêpe noire (Le Canard enchaîné, $n^{\circ} 4917$ du mercredi 21 janvier 2015, p. 6, Rue des Petites Perles).

(16) http://www.private-eye.co.uk/in-the-back, édition du 30 mars 2015.

(17) http://fr.wikipedia.org/wiki/Jeu_de_mots.

(18) Même source que 17. 
où le rire provient de l'homophonie entre " une crêpe ", la galette faite de farine, d'œufs et de lait, et " un crêpe ", le "brassard, insigne ou bande de drap noir porté ou arboré en signe de deuil(19) " ; et on imagine assez bien des joueurs de foot, une crêpe écrasée sur la figure...

On peut citer cet exemple (4) tiré de Private Eye (édition en ligne du 30 mars 2015) :

(4) TELEGRAPH'S APPLE CRUMBLE - Fawning coverage of Apple, and various fashion labels, shows advertising still rules at the Telegraph, despite editorials to the contrary.

où un amalgame est fait entre le dessert anglais, apple crumble, et la flagornerie des articles portant sur Apple, grand annonceur payant du Daily Telegraph.

D'autres procédés en langue anglaise sont également distingués tels les wellerisms(20), sorte d'anti-proverbes, formé sur le nom de Sam Weller, un des personnages du roman de Charles Dickens, Les Papiers posthumes du Pickwick Club ou The Pickwick Papers.

\subsection{Contrepèteries}

\subsubsection{Les Français ne font pas dans la dentelle}

La contrepèterie est définie par le Larousse comme "l'inversion de l'ordre des syllabes, des lettres ou des mots qui, modifiant le sens, produit des phrases burlesques ou grivoises(21)». Les exemples de contrepèteries issues du Canard enchaîné sont surtout grivois et se situent en général "en dessous de la ceinture ".

Dans Le Canard enchaîné, une rubrique regroupant les contrepèteries est publiée toutes les semaines: Sur l'album de la Comtesse(22) se trouve en page 7 du palmipède(23). Les trois exemples ci-dessous permettent de reconstituer un deuxième énoncé contenant les différents termes populaires et argotiques désignant un acte sexuel ou le sexe masculin(24) :

(5) Son Banier manque-t-il de pèse ? Le rigolo galéjait, se disant bien berné tout en l'abusant et palpait sans la moindre honte les chèques qu'il aimait. (Le Canard enchaîné du mercredi 4 février 2015, Sur l'album de la Comtesse, p. 7)

(6) Une railleuse a vu Sarko jouer des coudes : il tentait de forcer en paniquant dans les rangs de tête. (Le Canard enchaîné du mercredi 21 février 2015, Sur l'album de la Comtesse, p. 7).

(19) Les définitions proviennent du Larousse.

(20) Voici un exemple de wellerism : "Much noise and little wool," said the Devil when he sheared a pig.

(21) Les définitions sont issues du site www.larousse.fr/

(22) Luc Etienne, mathématicien, oulipien, régent au collège de Pataphysique, ami de la contrepèterie, fut "Comtesse " du Canard (entre 1957 et 1984) ; l'actuelle Comtesse est Joël Martin, écrivain.

(23) Palmipède est l'autre nom donné par ses collaborateurs au Canard enchaîné.

(24) L'usage veut qu'on ne donne jamais la solution d'une contrepèterie, chacun devant la trouver lui-même. Quelques indices sont toutefois donnés : gigolo et baise sont dans l'exemple (5) ; couille et forniquer dans l'exemple (6), alors que (7) contient deux synonymes de pénis. 
(7) Charlie indifférent à la peur qui mine, évoque les mythes sans Charb. (Le Canard enchaîné du mercredi 21 février 2015, Sur l'album de la Comtesse, p. 7).

Rabelais est associé aux premières contrepèteries dans la littérature française, deux exemples se trouvant dans Pantagruel en 1532(25). La contrepèterie disparaît en France pendant plusieurs siècles pour refaire surface au début du xxe siècle. Le Canard enchaîné est le seul hebdomadaire national à en publier plusieurs exemples toutes les semaines.

\subsubsection{Les Britanniques remettent le couvert}

Le terme générique anglais pour désigner la contrepèterie est spoonerism, défini comme "a humorous mistake in which a speaker switches the first sounds of two or more words "d'après le dictionnaire Merriam Webster ; ce terme a été fabriqué à partir du nom d'un révérend éducateur britannique du nom de William Spooner, enclin à ces erreurs involontaires dans ses sermons. Assimilée à une erreur ou mistake, la contrepèterie est à rapprocher de la dyslexie. De nos jours, l'erreur est devenue volontaire dans beaucoup d'exemples.

Private Eye a fabriqué un autre mot pour désigner ces mispronunciations ou malaproprisms, en les désignant du nom d'un présentateur sportif britannique, David Coleman, un habitué de ces linguistic balls-up(26) : les colemanballs(27) sont ainsi nées sous la plume des chroniqueurs du journal satirique et désignent " an inadvertent double-entendre or nonsensical statement broadcast on TV or radio ". Cependant, il n'y a pas de rubrique consacrée aux contrepèteries dans Private Eye.

Outre les malaproprisms, certains écrivains britanniques ont complété la liste des couverts commencée par le radical spoon, cuillère, en créant les forkerisms et les kniferisms, respectivement fourchette et couteau. II s'agit de nonce words, mots créés artificiellement, tel le mot supercalifragilisticexpialidocious(28) du film Mary Poppins (1964).

\subsection{Combinaisons et déformations}

Le troisième procédé est une combinaison des deux procédés précédents, c'est-à-dire une déformation lexicale d'expressions idiomatiques, de noms propres qui conservent toutefois leur " chaîne phonétique " et l'insertion d'une ou plusieurs syllabes créant une double lecture et faisant passer un message décalé.

(25) On y trouve la célèbre " folle à la messe " (molle à la fesse) ainsi que l'équivoque sur " À Beaumont-leVicomte " (à beau con le vit monte).

(26) Balls-up est le synonyme vulgaire de foul-up et désigne une gaffe ; la connotation sexuelle est explicite puisque le mot balls se traduit par " couilles".

(27) Source : www.albertjack.com/bookhomepages/samplepages/spoonerism.htm

(28) http://fr.wikipedia.org/wiki/Supercalifragilisticexpialidocious 
Le Canard enchaîné est passé maître dans cet art du mixage :

(8) Prophète l'humour... pas la guerre (Le Canard enchaîné $n^{\circ} 4917$ du mercredi 21 janvier 2015, p. 1)

(9) Pas Dieudonné à tout le monde (Le Canard enchaîné $n^{\circ} 4917$ du mercredi 21 janvier 2015, p. 8).

(10) A Davos, les riches vont l'avoir dans l'os (Le Canard enchaîné $n^{\circ} 4917$ du mercredi 21 janvier 2015, p. 8).

L'exemple (8) est bâti sur le dicton "Faites l'amour pas la guerre ", dans lequel la syllabe proest rajoutée au verbe Faites et donne Prophète par un jeu de mots portant sur l'homophonie ; humour est la déformation d'amour et l'arme de journaux satiriques, dont Charlie Hebdo qui a publié des caricatures du prophète Mahomet en 2006, provoquant la haine et les attentats du 7 janvier 2015.

Dans (9), le dicton populaire sur lequel repose l'effet burlesque est " c'est pas donné à tout le monde ", auquel on a rajouté le préfixe dieu-, pour faire référence au nom de l'humoriste controversé français Dieudonné.

(10) fonctionne sur un mode plus phonétique et crée une rime burlesque, à la façon d'une comptine pour les enfants.

Dans Private Eye, les exemples sont légion ; on peut citer :

(11) Lockerbie the flight from Justice (rapport spécial 2001).

(12) Giaka of all trades (rapport spécial 2001).

(13) Shady Arabia and the Desert Fix (numéro 1375 - 2 octobre 2014).

On a vu que les contrepèteries sont peu nombreuses dans Eye ; le mixage des effets linguistiques burlesques jouent ainsi sur d'autres registres. Ainsi dans (11), l'expression the fight for justice ou la recherche de la justice en français, est déformée en the flight from justice : le hors-série porte en effet sur l'attentat, dit de Lockerbie, non élucidé à ce jour contre le vol Pan Am 103 (flight) le 21 décembre 1988. La préposition from montre que la justice n'a pas été, ou risque de ne pas être rendue.

(12) joue sur le même registre du dicton anglais : Jack of all trades, master of none (Touche à tout, bon à rien) et remplace Jack par Giaka, prénom arabe d'un des agents libyens passés à la CIA.

(13) Shady Arabia, littéralement "Arabie pourrie ", se réfère phonétiquement à Saudi Arabia, Arabie Saoudite, et la coordonne à desert fix, qui est une déformation phonétique de desert fox, le fennec; fix peut avoir de nombreux sens dont celui de réparation ou de trucage. 
Après avoir décrit et étudié les trois procédés créant le rire dans ces journaux, nous allons proposer différentes traductions ou pistes de traduction pour nos exemples tirés du Canard et de Eye.

\section{Comparaison de quelques traductions}

\subsection{Fonction du journal satirique}

Comme indiqué ci-dessus, le journal satirique a une fonction informative sur un mode burlesque. Son lectorat étant très large, le journal doit communiquer en tenant compte des connaissances larges et générales de son lectorat ; son vocabulaire est non spécialisé et ses références sont populaires.

Le journal traite également de l'actualité qui est immédiate et éphémère.

Ces éléments doivent être pris en compte dans l'approche traductionnelle choisie.

Les extraits suivants proposent des jeux de mots qui sont identifiés et décrits dans le tableau ; les numéros des exemples sont suivis de " $a$ " ou de " $b$ "; le premier (a) renvoie à une stratégie de traduction stylistico-linguistique ; le second (b) renvoie à une stratégie fonctionnaliste.

\subsection{Jeux de mots et polysémie}

Les premiers exemples choisis portent sur la polysémie ; ces exemples sont diversement faciles à traduire.

\begin{tabular}{|l|l|}
\hline \multicolumn{1}{|c|}{$\begin{array}{c}\text { Canard enchaîné } \mathrm{n}^{\circ} 4917 \\
\text { mercredi } 21 \text { janvier } 2015\end{array}$} & \multicolumn{1}{c|}{ Traduction anglaise } \\
\hline $\begin{array}{l}\text { (14) après les attentats et les manifs, il s'envole } \\
\text { dans les sondages } \\
\text { Hollande : un président charlimastique }\end{array}$ & $\begin{array}{l}\text { (14a) } ?^{*} \text { After attempts and demonstrations, he } \\
\text { takes off in the polls } \\
\text { Hollande: a Charlimastic President }\end{array}$ \\
\hline $\begin{array}{l}\text { Jeu de mots et déformation : } \\
\text { charismatique devient charlimastique. }\end{array}$ & $\begin{array}{l}\text { (14b) After Charlie-Hebdo shooting and demons- } \\
\text { trations, his approval rating has skyrocketed } \\
\text { Hollande: a Charli-mastic President }\end{array}$ \\
\hline
\end{tabular}

(14a) est une traduction maladroite, voire douteuse et est signalée par ?* ; non idiomatique, on voit qu'il s'agit d'une traduction littérale (procédé 3 Vinay Dalbernet).

(14b) propose une traduction plus idiomatique : "s'envoler dans les sondages " est traduit par " sa côte de popularité culmine ", ce qui est la façon américaine ou anglaise de parler d'un sondage favorable à un homme politique ; le sens de la LC est pressenti pour la traduction. 


\begin{tabular}{|l|l|}
\hline \multicolumn{1}{|c|}{\begin{tabular}{c}
\multicolumn{1}{|c|}{ Canard enchaîné } \\
mercredi 18 février 2015, Une
\end{tabular}} & \multicolumn{1}{c|}{ Traduction anglaise } \\
\hline $\begin{array}{l}\text { (15) Procès du Carlton : les parties civiles se } \\
\text { désistent } \\
\text { "DSK enfin dans une bonne passe " }\end{array}$ & $\begin{array}{l}\text { (15 a) Carlton trial: the civil plaintiffs withdraw } \\
\text { DSK is finally in a good notch }\end{array}$ \\
\hline $\begin{array}{l}\text { Jeux de mots } \\
\text { Mots polysémiques : parties ; passe }\end{array}$ & $\begin{array}{l}\text { (15 b) France's Carlton trial: civil plaintiffs with- } \\
\text { draw } \\
\text { DSK slips out of trick-y position }\end{array}$ \\
\hline
\end{tabular}

(15) porte sur la polysémie entre les termes juridiques et des allusions sexuelles : parties et passe. (15a) propose une traduction littérale ; (15b) joue sur le mot trick qui signifie " passe " en anglais et position renvoie à Kama Sutra positions, ce qui est compréhensible dans la langue cible.

\begin{tabular}{|c|c|}
\hline $\begin{array}{c}\text { Canard enchaîné } \\
\text { mercredi } 4 \text { février } 2015 \text {, Une }\end{array}$ & \\
\hline $\begin{array}{l}\text { (16) La vie sexuelle de DSK étalée au tribunal de } \\
\text { Lille } \\
\text { Une affaire qui restera dans les anales! }\end{array}$ & $\begin{array}{l}\text { (16a) DSK's sexual life displayed in the Lille } \\
\text { Court: An affair to put down in history } \\
\text { To nail down in history }\end{array}$ \\
\hline Jeux de mots sur : affaire et anales & $\begin{array}{l}\text { (16b) DSK's sexcapades exposed at Lille court: } \\
\text { This case will change the intercourse of history! }\end{array}$ \\
\hline
\end{tabular}

(16a) est une traduction correcte mais qui ne rend pas tous les sens de anales.

(16b) rend le jeu de mots sur anales par un jeu alternatif : intercourse (rapport sexuel) utilisé à la place de course, ou cours (de l'histoire).

\begin{tabular}{|l|l|}
\hline \multicolumn{1}{|c|}{\begin{tabular}{c}
\multicolumn{1}{|c|}{ Canard enchaîné } \\
mercredi 4 février 2015, p. 3
\end{tabular}} & $\begin{array}{l}\text { (17a) A policewoman and Coulibaly's accomplice } \\
\text { were having a romance }\end{array}$ \\
\hline $\begin{array}{l}\text { (17) Une gendarmette et un complice de Couli- } \\
\text { baly s'aimaient d'amour tendre }\end{array}$ & $\begin{array}{l}\text { (17b) Love me tender: Lady cop was enamoured } \\
\text { of Coulibaly accomplice }\end{array}$ \\
\hline $\begin{array}{l}\text { Référence à la chanson de Juliette Gréco « Un } \\
\text { petit oiseau, un petit poisson " avec des paroles } \\
\text { de Boby Lapointe }\end{array}$ & \\
\hline
\end{tabular}

(17) fait référence à une chanson populaire française ; (17a) ne rend pas cette référence en anglais mais ne traduit que la référence en $\mathrm{LS}$ qui ne renvoie à aucune chanson en langue anglaise ; (17b) propose la chanson d'amour d'Elvis pour exprimer l'amour tendre entre la représentante de l'ordre public et le complice d'un terroriste. 


\subsection{Essais de traductions sur les contrepèteries}

\begin{tabular}{|l|l|}
\hline $\begin{array}{c}\text { Le Canard enchaîné du mercredi } 4 \text { février 2015, } \\
\text { Sur l'album de la Comtesse, p. 7 }\end{array}$ & \\
\hline (18) Son Banier manque-t-il de pèse? & $\begin{array}{l}\text { (18a) Is Banier short of cash? } \\
\text { (18b) Banier's assets in need of a hoist? }\end{array}$ \\
\hline $\begin{array}{l}\text { Le rigolo galéjait, se disant bien berné tout en } \\
\text { l'abusant et palpait sans la moindre honte les } \\
\text { chèques qu'il aimait }\end{array}$ & ??? \\
\hline
\end{tabular}

(18) est certainement l'un des procédés les plus difficiles à traduire car il joue sur les sonorités propres à chaque langue ; de plus l'élément culturel renvoie à une réalité culturelle unique ; il est fait référence au photographe François-Marie Banier et à l'affaire Bettencourt, du nom de Liliane Bettencourt, principale actionnaire de L'Oréal et première fortune de France.

\subsection{Procédés complexes}

Ces procédés sont les plus compliqués à traduire car ils combinent jeux de mots, expressions et déformations lexicales.

\begin{tabular}{|c|c|}
\hline $\begin{array}{l}\text { Le Canard enchaîné } \\
\text { du mercredi } 4 \text { février 2015, p. } 8\end{array}$ & \\
\hline $\begin{array}{l}\text { (19) Procès du Carlton : } \\
\text { pour les accusés, le box après le boxon! }\end{array}$ & $\begin{array}{l}\text { (19a) For the defendants, the dock after the bro- } \\
\text { thel }\end{array}$ \\
\hline $\begin{array}{l}\text { Box des accusés : dock } \\
\text { boxon : brothel }\end{array}$ & $\begin{array}{l}\text { (19b) Defendants go from whorehouse to court- } \\
\text { house }\end{array}$ \\
\hline
\end{tabular}

(19a) propose une traduction proche de la langue source, alors que (19b) utilise des composés formés sur house pour donner une lecture rythmée dans la langue cible.

\begin{tabular}{|c|c|}
\hline $\begin{array}{c}\text { Private Eye - } \\
\text { online version } 30 \text { March }\end{array}$ & \\
\hline (20) FIFA gets ready for a big sheikh-up & (20a) La FIFA prête au grand chamboulement \\
\hline
\end{tabular}

(20a) est une proposition de traduction ne rendant pas compte de l'élément humoristique de la langue source qui pourrait être transposable en langue cible. 
En effet, dans (20), il est difficile de trouver un mot français contenant le son " cheikh " et signifiant une grande transformation. La fréquence des sons n'étant pas semblable en français et en anglais, de même que la distribution des lettres (voyelles et consonnes), ce type de procédé, qui repose sur les sons, est particulièrement difficile à transposer.

\section{Conclusion}

Traduire le rire est un exercice linguistique complexe car il met en jeu de nombreux éléments lexicaux, stylistiques, sémantiques, phonétiques et culturels. Les jeux de mots reposant sur la polysémie sont les exercices de traduction les plus simples à rendre; et dès que le processus se complexifie, il devient difficile de traduire contrepèteries et autres mélanges d'effets de style et de sons.

Les approches envisagées se conçoivent comme des étapes successives et permettent de lever certaines ambiguïtés ; l'approche fonctionnaliste donne toutes les clés pour une traduction compréhensible en langue cible par les locuteurs ; cependant, des éléments mécaniques (sons et fréquence des combinaisons de lettres selon les langues) constituent un obstacle à cette traduction. Le traducteur se trouve dans une impasse et n'a pas d'autre choix que de recourir à une autre image :

(20) pourrait alors être traduit par : (20b) la FIFA s'offre un gros cheikh.

A défaut d'être fidèle, cette traduction remplit la même fonction communicationnelle(29) que la langue cible et pourrait être comprise et imprimée dans un journal satirique français comme le Canard enchaîné.

(29) Christiane Nord, p. 51. 


\section{Bibliographie}

CHUQUET, H., PAILLARD, M. 1989, Approche linguistique des problèmes de traduction. Paris : Ophrys.

LADMIRAL, Jean-René (2014) : Sourcier ou cibliste. Paris : Les Belles Lettres.

NORD, Christiane (1997/2008) : La Traduction : une activité ciblée. Introduction aux approches fonctionnalistes. (Traduit de l'anglais par Beverly Adab). Arras : Artois Presses Universitaires.

VINAY, Jean-Paul, DARBELNET, Jean (1958) : Stylistique comparée du français et de l'anglais. Paris : Didier.

Une du Canard enchaîné n 4921

Private Eye - special report

du 18 février 2015
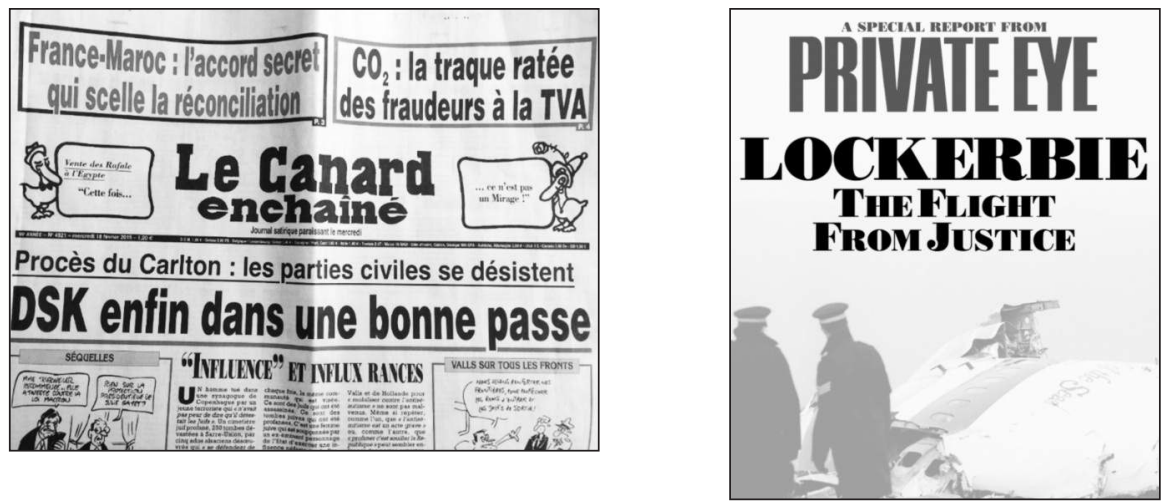

Joëlle Popineau est maître de conférences en anglais à l'université de Lorraine où elle enseigne la traduction économique en cycle Licence en Langues étrangères appliquées et la traduction spécialisée (anglais médical et juridique) en Master "Technologies de la traduction ".

Ses recherches et communications portent sur les différents aspects et approches de la traduction, qu'il s'agisse d'approches didactiques, linguistiques ou traductologiques.

Joëlle Popineau est membre associée de la SFT et membre consultatif d'Aprotrad de par son statut d'enseignante en traduction. 